\title{
AVALIAÇÃO DA QUALIDADE DE JUNTAS COLADAS DE SEIS ESPÉCIES DE MADEIRAS TROPICAIS DA AMAZÔNIA
}

\author{
Narciso Fernando Bila ${ }^{1}$, Setsuo Iwakiri2 ${ }^{2 *}$, Rosilani Trianoski², José Guilherme Prata ${ }^{2}$ \\ ${ }^{1}$ Universidade Eduardo Mondlane, Departamento de Engenharia Florestal, Maputo, Moçambique - narciso.bila@uem.mz \\ ${ }^{2}$ Universidade Federal do Paraná, Departamento de Engenharia e Tecnologia Florestal, Curitiba, Paraná, Brasil - setsuo@ufpr.br*; \\ rosilani@ufpr.br; jgprata@gmail.com
}

Recebido para a publicação: 28/04/2014 - Aceito para a publicação: 22/04/2016

\begin{abstract}
Resumo
Esse trabalho teve como objetivo avaliar a massa específica aparente, extrativos e a qualidade de juntas coladas de seis espécies de madeiras tropicais da Amazônia. As espécies estudadas foram Protium puncticulatum J. F. Macbr (Breu vermelho), Eschweilera coriácea (DC.) S.A. Mori (Piaozinho), Manilkara amazonica (Huber.) Standley (Abiurana), Inga paraensis Ducke (Inga vermelho), Eschweilera odora (Poepp.) Miers (Mata-Matá) e Byrsonima crispa Juss (Murici). A colagem foi realizada com os adesivos acetato de polivinila (PVAc) e Emulsão polimérica de isocianato(EPI) em gramaturas de $120 \mathrm{e} 200 \mathrm{~g} / \mathrm{m}^{2} \mathrm{e}$ aplicado em uma das faces de colagem. Os ensaios de cisalhamento da linha de cola e percentual de falhas na madeira foram realizados segundo as normas CEN/TS 13354:2003e ABNT-NBR ISO 12466-1:2006, respectivamente. Os resultados mostraram que todas as espécies estudadas apresentavam alta massa específica aparente e teor de extrativos totais de acordo com a literatura. Para as juntas coladas com adesivo PVAc, as madeiras de Eschweilera coriácea e Manilkara amazonica demostraram melhores resultados de cisalhamento. Para as juntas coladas com adesivo EPI, os melhores resultados foram obtidos para madeiras de Protium puncticulatum, Eschweilera coriácea, Inga paraensise Eschweilera odora. O adesivo EPI demostrou melhores resultados em relação ao PVAc. Não foram constatadas diferenças significativas entre as duas gramaturas empregadas. Com base nos requisítos mínimos estabelecidos pela norma CEN/TS 13354:2003, as espécies estudadas apresentaram potencial para produção de painéis EGP colados com adesivo EPI.
\end{abstract}

Palavras chave: EGP; acetato de polivinila; emulsão polimérica de isocianato.

\begin{abstract}
Evaluation of quality of the bonded joints of six species of amazonian tropical timbers. This study aimed to evaluate the apparent density, extractives and quality of bonded joints of six species of amazonian tropical timbers. The species studied were Protium puncticulatum JF Macbr (Breu vermelho), Eschweilera coriácea (DC.) S.A. Mori (Piaozinho), Manilkara amazonica (Huber.) Standley (Abiurana), Inga paraensis Ducke (Inga vermelho), Eschweilera odora (poepp.) Miers (Mata-Matá) and Byrsonima crispa Juss (Murici). The wood was bonded using polyvinyl acetate (PVAc) and polymeric emulsion of isocyanate (EPI) adhesives in weights of 120 and $200 \mathrm{~g} / \mathrm{m}^{2}$. Shear bondingtest and the wood failure percentage was performed in accordance with CEN/TS 13354:2003 and ABNT-NBR ISO 12466-1:2006 standards, respectively. The results showed that all species have high apparent densityand total extractives according to the literature. For the bonded joints with PVAc adhesive, Eschweilera coriacea and Manilkara amazonica showed high values of shear strength. For bonded joints with EPI adhesive, the best results were obtained for Protium puncticulatum, Eschweilera coriacea, Inga paraensis and Eschweilera odora. The EPI adhesive showed better results compared to PVAc. No significant differences between the two weights employed were found. Based on the minimum requirements established by CEN/TS 13354:2003 E, the studied species showed potential for producing EGP bonded with EPI adhesive.

Keywords: EGP; polyvinyl acetate; emulsion polymeric isocyanate.
\end{abstract}

\section{INTRODUÇÃO}

As florestas tropicais da região amazonica constituem um sistema de estrutura complexa que possui composição altamente diversificada de espécies com potencial madeireiro. Entretanto, o seu aproveitamento depende dos conhecimentos acerca das propriedades tecnológicas da madeira dessas espécies, visando o seu uso racional e sustentável, na geração de produtos de maior valor agregado.

Segundo a Sociedade Brasileira de Silvicultura (2006), a carência de informações consistentes sobre as espécies da floresta amazonica em relação às propriedades tecnológicas e seu desempenho em diferentes

FLORESTA, Curitiba, PR, v. 46, n. 4, p. 455 - 464, out. / dez. 2016.

Bila, N. F. et al.

ISSN eletrônico 1982-4688

DOI: $10.5380 /$ rf.v46i3.36311 
condições de uso, entre outros fatores, fazem com que as madeiras tropicais da Amazônia sejam ainda pouco utilizadas na produção indústrial.

Com a crescente demanda de produtos à base de madeira, há necessidade de desenvolvimento de pesquisas na área de tecnologia da madeira, visando obter benefícios econômicos provenientes de extração e processamento desse recurso, em consonância com os aspectos sociais e ambientais. As indústrias madeireiras da região amazonica produzem mecanicamente pisos de maderia maciça, serrados, lâminas e compensados. A utilização de resíduos provenientes de processamento industrial ainda é muito incipiente, resultando em desperdícios de madeiras que poderiam ser melhor aproveitadas. Uma das alternativas no aproveitamento de resíduos de processamento e de espécies comercialmente desconhecidas, mas com potencial tecnológico para uso industrial, seriam na fabricação de painéis de madeira sólida conhecidos como EGP (Edge glued panel).

O EGP consiste de sarrafos colados lateralmente, podendo estar unidos pelo topo através de emendas do tipo "finger joints", formando um painel. Várias indústrias já produzem os painéis EGP utilizando basicamente a madeira de florestas plantadas, principalmente as espécies de Pinus, Tectona grandis (teca) e em menor escala as madeiras de Eucalyptus grandis (PRATA, 2010).

Entretanto, para a fabricação de um produto colado, é importante que seja avaliada primeiramente a capacidade de adesão das madeiras utilizadas, pois, segundo Marra (1992), mais de 70\% de produtos a base de madeira utilizam algum tipo de adesivo na sua fabricação. A avaliação da qualidade de colagem de madeiras pode ser realizada por meio de ensaios de cisalhamento das juntas coladas e percentual de falhas da mesma no plano de colagem, com base nos procedimentos normatizados.

Perante o exposto, e partindo da hipótese que madeiras tropicais podem ser utilizadas para geração de produtos de maior valor agregado, esse trabalho teve como objetivo avaliar a massa específica aparante, o teor de extrativos e a resistência ao cisalhamento das juntas coladas de seis espécies de madeiras tropicais da Amazônia, visando inferir sobre o potencial dessas espécies na produção de painéis colados lateralmente (EGP).

\section{MATERIAL E MÉTODOS}

\section{Material}

Na presente pesquisa foram estudadas seis espécies de madeiras tropicais da Amazônia: Protium puncticulatum J.F. Macbr(Breu vermelho), Eschweilera coriácea (DC.) S.A. Mori (Piaozinho), Manilkara amazonica (Huber.) Standley (Abiurana), Inga paraensis Ducke (Inga vermelho), Eschweilera odora (poepp.) Miers (Mata-Matá) e Byrsonima crispa Juss (Murici). O material foi coletado na Estação Experimental ZF-2 do Instituto Nacional de Pesquisas da Amazônia, Manaus, Amazonas. Para a colagem das peças de madeira, foram utilizados os adesivos PVAc Aquence KL 245 D3 conhecido como Dorus KL 245A fabricado pela empresa Henkel Ltda., e o EPI EL-70 fabricado pela Momentive Química do Brasil Ltda.

\section{Método}

As madeiras das seis espécies foram obtidas na forma de tábuas, as quais foram secas, climatizadas e classificadas visualmente com base nos defeitos como nós, rachaduras e podridão. Das tábuas, foram confeccionados sarrafos com dimensões finais após aplainamento de 20 × 50 × $310 \mathrm{~mm}$ para espessura, largura e comprimento, sendo 16 sarrafos para cada espécie e totalizando 96 para as espécies estudadas.

A massa específica aparente $(12 \%)$ da madeira foi obtida a partir dos sarrafos preparados para a colagem, com base nos procedimentos descritos na norma ABNT 7190 (1997). O teor de extrativos totais e o pH das espécies foram determinados a partir de maravalhas obtidas do processo de usinagem dos sarrafos para confecção de juntas coladas, segundo as normas TAPPI 204 (2002) e TAPPI 252 (2002), respectivamente.

As juntas coladas foram confeccionadas com dois sarrafos colados aos pares, em função dos tratamentos pré-estabelecidos (espécie/adesivo/gramatura), sendo duas juntas por tratamento, conforme plano experimental apresentado na tabela 1 . O adesivo foi aplicado em uma das faces de colagem (linha simples) e espalhado com um rolo de espuma, em gramatura de 120 e $200 \mathrm{~g} / \mathrm{m}^{2}$, tanto para o PVAc, quanto para o EPI.

Para a colagem, com ambos os adesivos, foi empregada pressão específica de $6,45 \mathrm{kgf} / \mathrm{cm}^{2}$ exercida por meio de um torquímetro (30N.m), onde as peças permaneceram sob pressão por 3 horas à temperatura ambiente. Após a prensagem, as juntas coladas foram acondicionadas em câmera climatizada por um período de sete dias para cura total do adesivo antes da confecção dos corpos-de-prova.

De cada tratamento, foram selecionados 12 para os ensaios de cisalhamento para avaliação em condições de uso seco. Os ensaios foram conduzidos de acordo com os procedimentos descritos na norma CEN/TS 13354:2003, a qual estabelece que para as condições de uso a seco, os corpos-de-prova devem ser submetidos à imersão em água à temeperatura de $20^{\circ} \mathrm{C}$, por um período de 24 horas, antes dos ensaios. 
Os ensaios de cisalhamento foram realizados numa máquina de ensaios universal marca EMIC com capacidade para $300 \mathrm{kN}$, equipado com uma célula de carga com capacidade de 30kN. Após os ensaios, foi realizada a avaliação de percentagem de falhas na madeira, conforme a norma ABNT-NBR ISO 12466-1:2006. Para o atendimento das condições estabelecidas pela norma CEN/TS 13354:2003, foi determinado o quinto percentil inferior da resistência ao cisalhamento dos corpos-de-prova, calculado conforme a norma EN 326-1 (2002), o qual, deve ser igual ou superior a 2,5 MPa.

Tabela 1. Plano experimental.

Table 1. Experimental plan.

\begin{tabular}{|c|c|c|c|c|}
\hline Espécie & Adesivo & Gramatura $\left(g / \mathbf{m}^{2}\right)$ & $\mathrm{N}^{0}$ de juntas coladas & $\mathrm{N}^{0} \mathrm{CPs}$ \\
\hline \multirow{4}{*}{ Protium apiculatum } & \multirow{2}{*}{ PVAc } & 120 & 2 & 12 \\
\hline & & 200 & 2 & 12 \\
\hline & \multirow{2}{*}{ EPI } & 120 & 2 & 12 \\
\hline & & 200 & 2 & 12 \\
\hline \multirow{4}{*}{ Eschweilera coriácea } & \multirow{2}{*}{ PVAc } & 120 & 2 & 12 \\
\hline & & 200 & 2 & 12 \\
\hline & \multirow{2}{*}{ EPI } & 120 & 2 & 12 \\
\hline & & 200 & 2 & 12 \\
\hline \multirow{4}{*}{ Manilkara amazônica } & \multirow{2}{*}{ PVAc } & 120 & 2 & 12 \\
\hline & & 200 & 2 & 12 \\
\hline & \multirow{2}{*}{ EPI } & 120 & 2 & 12 \\
\hline & & 200 & 2 & 12 \\
\hline \multirow{4}{*}{ Inga paraenses } & \multirow{2}{*}{ PVAc } & 120 & 2 & 12 \\
\hline & & 200 & 2 & 12 \\
\hline & \multirow{2}{*}{ EPI } & 120 & 2 & 12 \\
\hline & & 200 & 2 & 12 \\
\hline \multirow{4}{*}{ Eschweilera odora } & \multirow{2}{*}{ PVAc } & 120 & 2 & 12 \\
\hline & & 200 & 2 & 12 \\
\hline & \multirow{2}{*}{ EPI } & 120 & 2 & 12 \\
\hline & & 200 & 2 & 12 \\
\hline \multirow{4}{*}{ Byrsonima crispa } & \multirow{2}{*}{ PVAc } & 120 & 2 & 12 \\
\hline & & 200 & 2 & 12 \\
\hline & \multirow{2}{*}{ EPI } & 120 & 2 & 12 \\
\hline & & 200 & 2 & 12 \\
\hline
\end{tabular}

CPs: Corpos de prova.

A análise estatística dos dados foi realizada por meio de um delineamento em arranjo fatorial (6 x 2 × 2$)$ com três variáveis (espécie, adesivo e gramatura). Inicialmente as variâncias dos tratamentos foram avaliadas quanto a sua homogeneidade pelo tesse de Bartlett e, em seguida, testadas pelo tesse $\mathrm{F}$ em análise de variância (ANOVA). Quando os resultados revelaram existir diferenças estatísticas significativas entre as médias dos tratamentos, as médias foram comparadas pelo tesse de Tukey ao nível de 5\% de probabilidade de erro.

\section{RESULTADOS E DISCUSSÃO}

\section{Massa específica aparente}

$\mathrm{Na}$ tabela 2, estão apresentados os resultados obtidos para massa específica aparente das madeiras de seis espécies estudadas. A espécie Eschweilera coriácea apresentou maior valor médio da massa específica aparente com $1,21 \mathrm{~g} / \mathrm{cm}^{3}$, e o menor valor médio foi obtido para espécie Inga paraensis com $0,81 \mathrm{~g} / \mathrm{cm}^{3}$. Não foi constatada diferença estatística entre os valores médios da massa específica aparente das espécies Eschweilera odora, Byrsonima crispa e Protium puncticulatum.

Tabela 2. Massa específica aparente das espécies estudadas.

Table 2. Apparent density of the studied species.

\begin{tabular}{lcc}
\hline Espécies & MEap $\left(\mathbf{g} / \mathbf{c m}^{\mathbf{3}}\right)$ & $\mathbf{C V}(\boldsymbol{\%})$ \\
\hline Eschweilera odora & $0,90 \mathrm{c}$ & 10,18 \\
Byrsonima crispa & $0,89 \mathrm{c}$ & 7,11 \\
Protium puncticulatum & $0,91 \mathrm{c}$ & 4,27 \\
Eschweilera coriácea & $1,21 \mathrm{a}$ & 1,28 \\
Manilkara amazonica & $1,13 \mathrm{~b}$ & 5,06 \\
Inga paraensis & $0,81 \mathrm{~d}$ & 7,53 \\
\hline
\end{tabular}

Médias seguidas pela mesma letra não diferem estatísticamente entre si ao nível de 5\% de probabilidade pelo Tesse de Tukey; MEap - massa específica aparente (12\%); CV- coeficiente de variação das amostras.

FLORESTA, Curitiba, PR, v. 46, n. 4, p. 455 - 464, out. / dez. 2016.

Bila, N. F. et al.

ISSN eletrônico 1982-4688

DOI: $10.5380 /$ rf.v46i3.36311 
Os valores obtidos para as espécies Eschweilera coriácea e Eschweilera odora avaliadas nesse estudo foram supeiores aos valores apresentados por Loureiro (1979) de 0,98 e 0,82 g/ $\mathrm{cm}^{3}$, respectivamente para as duas espécies. Essas diferenças podem ser atribuídas à variação que ocorre na massa específica aparente em função da procedência, idade, dentro e entre árvores da mesma espécie. Ressalta-se que para as demais espécies não foi encontrada bibliografia que menciona os valores da massa específica aparente das mesmas, não havendo portanto uma base de comparação.

Lisboa et al. (1993), determinaram o coeficiente de variação de massa espécífica aparente para espécies da região Amazônica e encontraram valor de referência de 10\%. Com base nessa informação, pode se afirmar que os coeficientes de variação da massa específica aparente obtidos para as seis espécies estudadas encontramse dentro do intervalo aceitável para os procedimentos experimentais.

Os valores de massa específica aparente das espécies estudadas nesse trabalho são maiores em comparação a algumas espécies tropicais comerciais como Angelim (Hymenolobium sp.), Tauari (Couratari guianensis), Amendoim (Pterogyne niteus),com valores de 0,79, 0,61 e 0,77 g/ $\mathrm{cm}^{3}$, respectivamente encontrados por Almeida (2013). Já, em relação às espécies Jatobá (Hymenaea courbaril), Muiracatiara (Astronium lecointei) e Cumarú (Dipteryx odorata),com massa específica aparente de 0,92,0,97 e 1,09 g/ $\mathrm{cm}^{3}$, respectivamente, estão dentro da faixa de valores entre 0,89 a $1,21 \mathrm{~g} / \mathrm{cm}^{3}$ obtidos para as seis espécies estudadas nesse trabalho.

Valores de massa específica aparente obtidos para as madeiras das espécies em estudo podem resultar em dificuldades de colagem para confecção de juntas coladas. De acordo com Burger e Richter (1991), madeiras de alta massa específica apresentam menor volume de espaços vazios para a penetração de adesivo líquido, acarretando na redução da resistência da ligação adesiva entre duas peças de madeira coladas. Sellers Jr. (1994) afirma que o uso de madeiras de maior massa específica requer melhor qualidade do adesivo e controle dos procedimentos de colagem.

\section{Teor de extrativos totais e pH}

$\mathrm{Na}$ tabela 3, estão apresentados os resultados de teor de extrativos totais e pH das seis espécies estudadas. O maior valor médio do teor de extrativos totais foi obtido para Protium puncticulatum com 11,67\% e o menor valor para Byrsonima crispa com 3,19\%. As espécies Eschweilera odora, Eschweilera coriácea, Manilkara amazonica e Inga paraensisnão diferiram estatísticamente entre si, com valores intermediários compreendidos entre 7,68 e $9,24 \%$.

Tabela 3. Valores médios de teor de extrativos totais e $\mathrm{pH}$ das espécies.

Table 3. Mean value of extractives content and $\mathrm{pH}$ of the species.

\begin{tabular}{lcc}
\hline Espécies & Extrativos totais $\mathbf{( \% )}$ & $\mathbf{p H}(\boldsymbol{\%})$ \\
\hline \multirow{2}{*}{ Eschweilera odora } & $7,68 \mathrm{c}$ & $4,69 \mathrm{~b}$ \\
& $(0,82)$ & $(2,36)$ \\
Inga paraensis & $8,36 \mathrm{~b} \mathrm{c}$ & $5,24 \mathrm{a}$ \\
& $(7,72)$ & $(1,19)$ \\
Eschweilera coriácea & $7,79 \mathrm{c}$ & $4,91 \mathrm{~b}$ \\
& $(4,26)$ & $(5,52)$ \\
Manilkara amazonica & $9,24 \mathrm{~b}$ & $4,92 \mathrm{ab}$ \\
& $(5,82)$ & $(3,35)$ \\
Byrsonima crispa & $3,19 \mathrm{~d}$ & $3,48 \mathrm{~d}$ \\
\multirow{2}{*}{ Protium puncticulatum } & $(3,07)$ & $(1,67)$ \\
& $11,67 \mathrm{a}$ & $4,36 \mathrm{c}$ \\
\end{tabular}

Médias seguidas pela mesma letra não diferem estatísticamente entre si ao nível de 5\% de probabilidade pelo Tesse de Tukey; Os valores entre parêntesis correspondem coeficiente de variação.

Com exceção da espécie Protium puncticulatum, os valores médios de teor de extrativos totais encontrados para todas as espécies estudadas foram menores que $10 \%$. Esse fato é importante, tendo em vista a utilização dessas espécies na fabricação de painéis EGP, pois, Jankowsky (1988), afirma que espécies de madeiras tropicais com valores superiores a $10 \%$ podem apresentar dificuldades de colagem.

Os valores médios de $\mathrm{pH}$ variaram na faixa de 3,48 a 5,24, sendo o menor valor obtido para Byrsonima crispa e maior valor para Inga paraensis. Os valores médios de $\mathrm{pH}$ das espécies Eschweilera odora, Manilkara amazonica, Eschweilera coriácea e Inga paraensis, foram estatísticamente iguais entre si e superiores às demais. De acordo com Kollmann et al. (1975); Iwakiri (2005), espécies com baixo pH podem dificultar o processo de colagem para fabricação de produtos de madeiras. Com relação aos valores apresentados na literatura, as médias de pH obtidas para as seis espécies estudadas encontram-se dentro da faixa citada por Stamm (1964), entre 3,0 e 5,5. 


\section{Resistência das juntas coladas aos ensaios de cisalhamento}

$\mathrm{Na}$ tabela 4 estão apresentados as médias de tensão máxima de cisalhamento, percentual de falhas na madeira e $5^{\circ}$ percentil inferior, obtidos para cada espécie, em função do adesivo e gramatura, após prétratamento dos corpos-de-prova para uso em condição seca. Os valores de tensão máxima de cisalhamento e do $5^{\circ}$ percentil inferior, destacados em negrito, atendem os requisítos mínimos da norma CEN/TS 13354:2003, e, por conseguinte, as espécies abrangidas nessas condições são recomendadas para a produção de EGP.

Para as juntas coladas com o adesivo PVAc, em ambas as gramaturas, as espécies Eschweilera coriácea e Manilkara amazonica atenderam aos requisitos mínimos exigidos pela norma. Por outro lado, as espécies Protium puncticulatume Eschweilera odora apresentaram boa colagem das juntas coladas apenas em gramaturas de 200 e $120 \mathrm{~g} / \mathrm{m}^{2}$, respectivamente. As espécies Inga paraensis e Byrsonima crispa não atenderam aos requisítos mínimos estabelecidos pela norma CEN/TS 13354:2003.

Tabela 4. Resistência das juntas coladas ao cisalhamento.

Table 4. Shear strength of glued joints.

\begin{tabular}{|c|c|c|c|c|c|c|}
\hline Espécie & Adesivo & $\begin{array}{c}\text { Gramatura } \\
\left(\mathrm{g} / \mathrm{m}^{2}\right)\end{array}$ & $\begin{array}{l}\mathbf{F}_{\mathbf{M}} \\
(\%)\end{array}$ & $\begin{array}{c}\text { TC } \\
(\mathrm{MPa}) \\
\end{array}$ & $\begin{array}{l}\mathbf{C V} \\
(\%) \\
\end{array}$ & $\begin{array}{l}5^{\circ} \text { Percentil inferior } \\
(\mathrm{MPa})\end{array}$ \\
\hline \multirow{4}{*}{$\begin{array}{l}\text { Protium } \\
\text { puncticulatum }\end{array}$} & \multirow{2}{*}{ PVAc } & 120 & 0 & 3,19 & 39,67 & 1,45 \\
\hline & & 200 & 0 & 4,14 & 25,12 & 2,72 \\
\hline & \multirow{2}{*}{ EPI } & 120 & 5,83 & 9,72 & 19,15 & 6,33 \\
\hline & & 200 & 33,33 & 11,77 & 12,21 & 9,80 \\
\hline \multirow{4}{*}{$\begin{array}{l}\text { Eschweilera } \\
\text { coriácea }\end{array}$} & \multirow{2}{*}{ PVAc } & 120 & 0 & 7,23 & 20,75 & 4,97 \\
\hline & & 200 & 0 & 6,91 & 18,73 & 5,05 \\
\hline & \multirow{2}{*}{ EPI } & 120 & 15,00 & 14,38 & 21,78 & 9,63 \\
\hline & & 200 & 8,75 & 13,76 & 33,79 & 6,65 \\
\hline \multirow{4}{*}{$\begin{array}{l}\text { Manilkara } \\
\text { amazonica }\end{array}$} & \multirow{2}{*}{ PVAc } & 120 & 0 & 8,45 & 31,18 & 4,77 \\
\hline & & 200 & 0 & 7,43 & 31,04 & 3,13 \\
\hline & \multirow{2}{*}{ EPI } & 120 & 17,50 & 9,72 & 29,77 & 5,35 \\
\hline & & 200 & 3,33 & 7,66 & 46,30 & 2,66 \\
\hline \multirow{4}{*}{ Inga paraensis } & \multirow{2}{*}{ PVAc } & 120 & 0 & 2,46 & 64,13 & 0,41 \\
\hline & & 200 & 0 & 4,07 & 29,13 & 2,33 \\
\hline & \multirow{2}{*}{ EPI } & 120 & 5,00 & 7,92 & 18,18 & 5,77 \\
\hline & & 200 & 12,08 & 10,78 & 9,29 & 9,16 \\
\hline \multirow{4}{*}{$\begin{array}{l}\text { Eschweilera } \\
\text { odora }\end{array}$} & \multirow{2}{*}{ PVAc } & 120 & 0 & 4,13 & 17,79 & 3,15 \\
\hline & & 200 & 0 & 3,73 & 31,30 & 1,82 \\
\hline & \multirow{2}{*}{ EPI } & 120 & 19,17 & 9,78 & 16,86 & 7,10 \\
\hline & & 200 & 22,50 & 11,54 & 17,08 & 9,22 \\
\hline \multirow{4}{*}{$\begin{array}{l}\text { Byrsonima } \\
\text { crispa }\end{array}$} & \multirow{2}{*}{ PVAc } & 120 & 0 & 2,24 & 43,62 & 0,93 \\
\hline & & 200 & 0 & 3,14 & 24,62 & 2,06 \\
\hline & \multirow{2}{*}{ EPI } & 120 & 10,42 & 6,85 & 60,82 & 2,28 \\
\hline & & 200 & 18,75 & 8,45 & 25,84 & 5,52 \\
\hline
\end{tabular}

$\mathrm{F}_{\mathrm{M}}$ - porcentagem de falha na madeira; TC - Tensão máxima de cisalhamento; $\mathrm{CV}$ - coeficiente de variação; valores em negrito atenderam os requisitos da norma CEN/TS 13354:2003 E (5 percentil $\geq 2,5 \mathrm{MPa})$.

Para as juntas coladas com o adesivo EPI, em ambas as gramaturas, as espécies Protium puncticulatum, Eschweilera coriácea, Inga paraensis, Manilkara amazonica e Eschweilera odora atenderam as exigências da norma CEN/TS 13354:2003. A espécie Byrsonima crispa apresentou boa colagem somente na gramatura de $200 \mathrm{~g} / \mathrm{m}^{2}$.

Em relação ao percentual de falhas na madeira, todas as espécies estudadas quando coladas com adesivo PVAc apresentaram 0\%, ou seja, nenhuma fibra foi arrancada nas faces coladas dos corpos de prova. Esse resultado demonstra a baixa resistência do adesivo a base de PVAc em contato direto com a água. Já, para as juntas coladas com o adesivo EPI, verificaram se falhas na madeira, mas em percentual abaixo de $50 \%$.

Ressalta-se que, o percentual de falha na madeira entre $0 \%$ - 25\% indica baixa resistência do adesivo e/ou deficiência no processo de colagem tais como superficie lisa, baixa gramatura aplicada, alta umidade na superfície, entre outros; entre 50\% - 70\% evidencia uma situação mais adequada do processo de colagem e propriedades do adesivo, e entre $75 \%$ e $100 \%$, propriedades de adesão mais fortes (MARRA, 1992). 
De forma geral, os resultados apresentados na tabela 4 indicam melhor comportamento do adesivo EPI em relação ao PVAc, tendo em vista que o atendimento aos requisitos da norma CEN/TS 13354:2003, e, todas as espécies apresentaram falhas na madeira, embora com baixo percentual.

As três variáveis avaliadas nessa pesquisa (espécie, adesivo e gramatura) se mostraram significativos com relação à resistência ao cisalhamento das juntas coladas, onde as análises subsequentes desse trabalho estão voltadas a discussão individual das mesmas.

\section{Influência da espécie na resistência das juntas coladas}

Na tabela 5 estão apresentados os resultados de ensaios de cisalhamento das juntas coladas com adesivo PVAc, nas duas gramaturas, para as seis espécies estuadadas.

Tabela 5. Resistência das juntas coladas das seis espécies com adesivo PVAc.

Table 5. Shear strength of glued joints with PVAc for six species.

\begin{tabular}{llll}
\hline \multirow{2}{*}{ Adesivo } & Espécie & Gramatura $\left(\mathbf{g} / \mathbf{m}^{\mathbf{2}}\right)$ & \\
& Eschweilera coriácea & $\mathbf{1 2 0} / \mathbf{T C}(\mathbf{M P a})$ & $\mathbf{2 0 0 / T C} \mathbf{( M P a})$ \\
\hline \multirow{2}{*}{ PVAc } & Manilkara amazonica & $7,23 \mathrm{a}$ & $6,91 \mathrm{a}$ \\
& Protium puncticulatum & $8,45 \mathrm{a}$ & $7,43 \mathrm{a}$ \\
& Inga paraensis & $3,19 \mathrm{bc}$ & $4,14 \mathrm{~b}$ \\
& Eschweilera odora & $2,46 \mathrm{bc}$ & $4,07 \mathrm{~b}$ \\
& Byrsonima crispa & $4,13 \mathrm{~b}$ & $3,73 \mathrm{~b}$ \\
& $2,24 \mathrm{c}$ & $3,14 \mathrm{~b}$ \\
\hline
\end{tabular}

Médias seguidas pela mesma letra na mesma coluna não diferem estatísticamente entre si ao nível de 5\% de probabilidade. TC: tensão de cisalhamento.

Para as duas gramaturas, as espécies Eschweilera coriácea e Manilkara amazonica apresentaram valores de tensão de cisalhamento estatísticamente superiores em relação às demais apesar de serem muito densas. Esse fato não está em conformidade com os postulados de Vick (1999) e Iwakiri (2005), entre outros autores, que afirmam que, espécies com elevada massa específica são mais difíceis de serem coladas devido a paredes celulares mais espessas e lúmens pequenos, dificulatando a penetração do adesivo no processo de colagem.

O teor de extrativos totais das espécies não influenciou de forma clara na resistência da ligação adesiva das juntas coladas. As espécies Protium puncticulatum e Byrsonia crispa apresentaram respectivamente maior e menor teor de extrativos totais, entretanto, ambas as espécies apresentaram menores valores de tensão de cisalhamento. Portanto, os resultados obtidos contrariam a teoria relatada por Moslemi (1974), de que o elevado teor de extrativos pode reduzir consideravelmente a resistência da ligação adesiva.

Na tabela 6 estão apresentados os resultados de ensaios de cisalhamento das juntas coladas com adesivo EPI, nas duas gramaturas, para as seis espécies estuadadas. Não foram observadas relações clarassobre a influência da massa específica aparente, teor de extrativos totais e pH na resistência da ligação adesiva das juntas coladas de seis espécies estudadas.

Tabela 6. Resistência das juntas coladas das seis espécies coladas com adesivo EPI.

Table 6. Shear strength of glued joints with EPI for six species.

\begin{tabular}{llcc}
\hline \multirow{2}{*}{ Adesivo } & \multicolumn{1}{c}{ Espécie } & \multicolumn{2}{c}{ Gramatura $\left(\mathbf{g} / \mathbf{m}^{\mathbf{2}}\right)$} \\
& Protium puncticulatum & $9,72 \mathrm{~b} / \mathbf{T C}$ (MPa) & $\mathbf{2 0 0 / T C}(\mathbf{M P a})$ \\
\hline \multirow{4}{*}{ EPI } & Eschweilera odora & $9,78 \mathrm{~b}$ & $11,77 \mathrm{a}$ \\
& Manilkara amazonica & $9,72 \mathrm{~b}$ & $11,54 \mathrm{ab}$ \\
& Eschweilera coriácea & $14,38 \mathrm{a}$ & $7,66 \mathrm{c}$ \\
& Inga paraensis & $7,92 \mathrm{bc}$ & $13,76 \mathrm{a}$ \\
& Byrsonima crispa & $6,85 \mathrm{c}$ & $10,78 \mathrm{abc}$ \\
\end{tabular}

Médias seguidas pela mesma letra na mesma coluna não diferem estatisticamente entre si ao nível de 5\% de probabilidade.

\section{Influência do adesivo na resistência das juntas coladas}

Na tabela 7 estão apresentados os resultados de ensaios de cisalhamento das juntas coladas com adesivo PVAc e EPI, para as seis espécies estudadas.

Com exceção da espécie Manilkara amazonica, para todas as demais espécies estudadas as juntas coladas com o adesivo EPI apresentaram valores médios de cisalhamento estatísticamente superiores em relação às juntas coladas com o adesivo PVAc, tanto para a gramatura de $120 \mathrm{~g} / \mathrm{m}^{2}$, quanto para $200 \mathrm{~g} / \mathrm{m}^{2}$. 
Tabela 7. Resistência das juntas coladas com adesivo PVAc e EPI.

Table 7. Shear strength of glued joints with PVAc and EPI adhesives.

\begin{tabular}{llcc}
\hline \multirow{2}{*}{ Espécie } & \multicolumn{1}{c}{ Adesivo } & \multicolumn{2}{c}{ Gramatura $\left(\mathbf{g} / \mathbf{m}^{\mathbf{2}}\right)$} \\
& & $\mathbf{1 2 0} / \mathbf{T C}(\mathbf{M P a})$ & $\mathbf{2 0 0 / T C}(\mathbf{M P a})$ \\
\hline \multirow{2}{*}{ Protium puncticulatum } & PVAc & $3,19 \mathrm{~b}$ & $4,14 \mathrm{~b}$ \\
& EPI & $9,72 \mathrm{a}$ & $11,77 \mathrm{a}$ \\
\hline \multirow{2}{*}{ Eschweilera coriácea } & PVAc & $7,23 \mathrm{~b}$ & $6,91 \mathrm{~b}$ \\
& EPI & $14,38 \mathrm{a}$ & $13,76 \mathrm{a}$ \\
\hline \multirow{2}{*}{ Manilkara amazonica } & PVAc & $8,45 \mathrm{a}$ & $7,43 \mathrm{a}$ \\
& EPI & $9,72 \mathrm{a}$ & $7,66 \mathrm{a}$ \\
\hline \multirow{2}{*}{ Inga paraensis } & PVAc & $2,46 \mathrm{~b}$ & $4,07 \mathrm{~b}$ \\
& EPI & $7,92 \mathrm{a}$ & $10,78 \mathrm{a}$ \\
\hline \multirow{2}{*}{ Eschweilera odora } & PVAc & $4,13 \mathrm{~b}$ & $3,73 \mathrm{~b}$ \\
\multirow{2}{*}{ Byrsonima crispa } & EPI & $9,78 \mathrm{a}$ & $11,54 \mathrm{a}$ \\
\hline
\end{tabular}

Médias seguidas pela mesma letra na coluna na mesma linha não diferem estatísticamente entre si ao nível de 5\% de probabilidade.

O baixo desempenho das juntas coladas com PVAc em comparação ao adesivo EPI, pode ser atribuido a forma como se procede a cura desses adesivos,pois, de acordo com Correa (1997), a cura do adesivo PVAc ocorre pela simples evaporação do solvente (água), resultando numa colagem de baixa resistência quando expostas às condições de alta umidade. Já a cura do adesivo EPI ocorre pela reação de seus grupos de isocianato com os grupos hidroxilas da madeira, promovendo uma adesão química mais forte. Cabe ressaltar que, na presente pesquisa os ensaios foram realizados após pré-tratamento em água fria por 24 horas, condições essas, desfavoráveis ao adesivo PVAc.

$\mathrm{Na}$ analise da resistência ao cisalhamento de painéis EGP de madeira tropical e Eucalyptus benthamii produzidos com adesivo EPI, poliuretano e PVAc, Martins (2011) e Almeida (2013), verificaram melhor desempenho dos adesivos EPI e poliuretano.

\section{Influência da gramatura na resistência das juntas coladas}

$\mathrm{Na}$ tabela 8 estão apresentados os resultados de ensaios de cisalhamento das juntas coladas com adesivo PVAc e EPI, para diferentes gramaturas.

Tabela 8. Resistência das juntas coladas com duas diferentes gramaturas.

Table 8. Shear strength of glued joints with two different weights.

\begin{tabular}{llccccc}
\hline \multirow{2}{*}{$\begin{array}{l}\text { Gramatura } \\
\left(\mathbf{g} / \mathbf{m}^{\mathbf{2}}\right)\end{array}$} & Tensão (MPa) & $\mathbf{F}$ & $p$ & Tensão (MPa) & F & $p$ \\
\hline 120 & $4,615 \mathrm{a}$ & $0,4732^{\text {ns }}$ & 0,051 & $\begin{array}{r}9,73 \mathrm{a} \\
10,66 \mathrm{a}\end{array}$ & $2,80^{\text {ns }}$ & 0,048 \\
\cline { 2 - 7 } & $4,901 \mathrm{a}$ & & & &
\end{tabular}

ns - não significativo ( $>$ > 0,05); Médias seguidas seguidas pela mesma letra na coluna não diferem estatísticamente entre si. Foi aplicado o tesse Tukey ao nível de $5 \%$ de probabilidade.

Tanto para o adesivo PVAc, quanto para o EPI, não foram constatadas diferenças estatísticas para os valores médios de cisalhamento das juntas coladas com gramatura de $120 \mathrm{~g} / \mathrm{m}^{2}$ e de $200 \mathrm{~g} / \mathrm{m}^{2}$.

Nos ensaios de cisalhamento de painéis EGP de madeiras tropicais produzidos com adesivo PVAc e EPI, com gramaturas de 150 e $200 \mathrm{~g} / \mathrm{m}^{2}$, Almeida (2013) também não encontrou diferenças estatisicas. Da mesma forma, Prata (2010) não encontrou diferenças significativas entre as gramaturas de 150 e $200 \mathrm{~g} / \mathrm{m}^{2} \mathrm{nos}$ ensaios de cisalhamento das juntas coladas de madeira de Pinus tropicais com adesivo PVAc.

Os resultados obtidos nessa pesquisa e os apresentados na literatura, indicam a possibilidade de produção de painéis EGP com gramaturas menores, tendo em vistapropiciar a economia no consumo de adesivo sem comprometer a resistência da ligação adesiva das peças coladas.

\section{CONCLUSÕES}

- Maiores valores de massa específica aparente foram obtidos para as espécies Eschweilera coriácea e Manilkara amazonica e o menor valor para a espécie Inga paraensis. 
- Os valores de $\mathrm{pH}$ e teor de extrativos totaisobtidos para as seis espécies estudadas estão dentro da faixa de valores apresentados na literatura.

- As espécies Eschweilera coriácea e Manilkara amazonica apresentaram melhores resultados de resistência das juntas coladas em comparação às demais espécies.

- O adesivo EPI apresentou melhores resultados de resistência das juntas coladas em comparação ao adesivo PVAc.

- O aumento na gramatura de 120 para $200 \mathrm{~g} / \mathrm{m}^{2}$, não contribuiu para o aumento nos valores de tensão de cisalhamento das juntas coladas.

- Com exceção das juntas coladas com as espécies Inga paraensis e Byrsonima crispa, com adesivo PVAc, todas as demais espécies atenderam aos requisitos da norma CEN/TS 13354:2003. Para as juntas coladas com o adesivo EPI, todas as espécies seguiram aos requisitos da referida norma. Portanto, pode-se afirmar que as espécies estudadas nessa pesquisa possuem potencial para produção de painéis colados lateralmente (EGP).

\section{AGRADECIMENTOS}

Os autores expressam seus agradecimentos ao INCT/INPA - Madeiras da Amazônia, às empresas Henkel e Momentive, pela doação da madeira e adesivos utilizados nessa pesquisa.

\section{REFERÊNCIAS}

ALMEIDA, V. C. Avaliação do potencial de uso de resíduos de madeira tropical para produção de painéis colados lateralmente - EGP. Dissertação (Dotourado em Engenharia Florestal). Universidade Federal de Paraná, Curitiba 2013. 122 p.

ASSOCIAÇÃO BRASILEIRA DE NORMAS TÉCNICAS (ABNT). Projeto de estruturas de madeira NBR 7190. 107 p. 1997.

Madeira compensada - Qualidade de colagem. NBR ISO 12466-1. 2006.

BURGER, L. M.; RICHTER, H. G. Anatomia da Madeira. São Paulo. Nobel. 1991. 154 p.

CEN/TS 13354 - European committee for standardization. Solid wood panel -bonding quality -test method. Technical specification, May 2003.

CORRÊA, C. Técnicas de Colegam de Madeira com adesivos Vinílicos. Borden. Química Indústria e comércio Ltda, 1997. 68 p.

EUROPEAN STANDARD. EN 326-1: Wood-based panels - sampling, cutting and inspection - Part 1: Sampling and cutting of test pieces and expression of test results. 7 p. Bruxelas, 2002.

EUROPEAN STANDARD. EN 13354: Solid Wood panels (SWP) - bonding quality. Bruxelas, 2008. 10 p.

IWAKIRI, S. Painéis de Madeira reconstituída. FUPEF. 247 p. Curitiba. 2005.

JANKOWSKY, I. P. Colagem de madeiras. Piracicaba: ESALQ, 45 p. 1988.

KLEPPE, P. J. Forest Product Journal, Madison, v. 20, n. 5, p. 50-59, 1970.

KOLLMANN, F.; KENZI, P.; STAMM, A.; Principles of wood science and technology II. Wood based materials, 703 p. New York, USA, 1975.

LISBOA, C. D. J.; MATOS, J. L.; MELO, J. E. Amostragem e Propriedades Físico-Mecânicas de Madeiras Amazonicas. Colecção Meio Ambiente. Série Estudos - Floresta, nº 1. Brasilia, 1993. 103 p.

LOUREIRO, A. A.; SIlVA, M. F.; ALENCAR, J. C. Essências madeireiras da Amazônia. Vol. II. INPA/SUFRAMA, Manaus 1979.

MARRA, A. A., Technology of wood bonding. Nova York: Van Nostrand Reinhold, 1992. 453 p.

MARTINS, S. A. Adequação tecnológica da madeira de Eucalyptus benthamii Maiden et Cambage para produção de painéis colados laterralmente (PCL). Dissertação (Mestrado em ciências Florestais) Universidade de Brasília, Brasília/DF, 2011. 114 p. 
MOSLEMI, A. A. Particleboard. London: Southern Illinois University Press, 1974. 245 p.

PRATA, J. G. Estudo da viabilidade tecnológica do uso de espécies de Pinus tropicais para produção de painéis colados lateralmente (Edge glued panels - EGP). Tese (Doutorado em Engenharia Florestal). Universidade Federal do Paraná, Curitiba, 2010. 107 p.

SELLERS JR., T. Adhesive in the Wood Industry. In: PIZZI. A.; MITTAL, K. L. Handbook of adhesive technology. New York: Marcel Dekker, 1994. cap. 37.

SOCIEDADE BRASILEIRA DE SILVICULTURA (SBS). Fatos e números do Brasil florestal. nov. 2006, 107 p. Disponível em: < http://www.ipef.br/estatísticas/relatorios/ SBS-2005.pdf>. Acesso em: 14 Out. 2012.

STAMM, A. J. Wood and cellulose science. The Ronald Press Company - New York, 1964, 549 p.

TECHNICAL ASSOCIATION OF THE PULP AND PAPER INDUSTRY. TAPPI 204. Solvent extractives of wood and. Atlanta, 2002. Atlanta, 2002.

TAPPI 252. $\mathrm{pH}$ and electrical conductivity of hot water extracts of pulp, paper, and paperboard.

VICK, C. B. Adhesive Bonding of Wood Materials. U. S. PRODUCTS LABORATORY. Wood handbook Wood as an engineering material. Gen. Tech. Rep. FPL-GTR-113. Department of Agriculture, Forest Service, Forest Products Laboratory. Chapter 9. 463 p. Madison: U.S. 1999. 